\title{
Pricing Holder-Extendable Call Options with Mean-Reverting Stochastic Volatility
}

\author{
S.N.I. IBRAHIM ${ }^{\square}{ }^{1,2}$, A. DIAZ-HERNANDEZ ${ }^{3}$, J.G. O'HARA ${ }^{4}$ and N. CONSTANTINOU ${ }^{5}$
}

(Received xx Month 2019)

\begin{abstract}
Options with extendable features have many applications in finance which provides the motivation for this study. The price of extendable options has appeared in the literature when the underlying asset follows a geometric Brownian motion (GBM) with a constant volatility. In this paper, we consider holder-extendable call options when the underlying asset follows a mean-reverting stochastic volatility. The option price is expressed in integral forms which have known closed-form characteristic functions. We price these options using fast Fourier transform (FFT), finite difference method, and Monte Carlo simulation, and determine the efficiency and accuracy of the Fourier method in pricing holder-extendable call options for Heston parameters calibrated to the sub-prime crisis. We show that the FFT reduces the computational time required to produce a range of holder-extendable call option prices by at least an order of magnitude. Numerical results also demonstrate that when the Heston correlation is negative, Black-Scholes underprices in-the-money and over-prices out-of-the-money holder-extendable call options compared to the Heston model, which is analogous to the behaviour for vanilla calls.
\end{abstract}

2010 Mathematics subject classification: 91G20.

Keywords and phrases: extendable options, Heston model, fast Fourier transform, finite difference method, Monte Carlo simulation.

\footnotetext{
${ }^{1}$ Department of Mathematics, Faculty of Science, Universiti Putra Malaysia, 43400 UPM Serdang, Selangor, Malaysia; e-mail: iqmal@upm.edu.my http://orcid.org/0000-0003-0798-5810 orcid:0000-0003-0798-5810.

${ }^{2}$ Institute for Mathematical Research, Universiti Putra Malaysia, 43400 UPM Serdang, Selangor, Malaysia.

${ }^{3}$ Faculty of Economics and Business, Universidad Anahuac Mexico-Norte, 52786, Mexico; e-mail: adan.diaz@anahuac.mx http://orcid.org/0000-0001-5571-8157 orcid:0000-0001-55718157.

${ }^{4}$ School of Computer Science and Electronic Engineering, University of Essex, Colchester CO4 3SQ, United Kingdom; e-mail: johara@essex.ac.uk http://orcid.org/0000-0001-6097-1130 orcid:0000-0001-6097-1130.

${ }^{5}$ Deceased.
} 


\section{Introduction}

As Longstaff [22] has described, "Any financial contract that could involve a rescheduling of payments, a renegotiation of terms, an early call or exercise provision or some similar type of flexibility over the timing of cash flows could be viewed generally as including an option with an extendable maturity". One such option is the extendable option which can either be a holder-extendable option or a writerextendable option, other than being classified as calls and puts. At the initial maturity time, the holder-extendable option can be extended to another maturity time for an additional premium, whereas the writer-extendable option can be extended to another maturity time if the option is out-of-the-money. The pricing for the writer-extendable option is more straightforward than the holder-extendable option; hence is not included in this study.

An extendible option is an example of a dual-expiry exotic option [4], and its framework has been used in other financial contracts such as extendable warrants [13, 16] and extendable bonds [25]. In addition, Longstaff [22] discusses other applications of extendable options to real-estate and shared-equity mortgages. Hauser and Lauterbach [13] suggest that investors are favorable of extendable call warrants because they produce lower absolute pricing errors than the standard call warrants. Extendable options are also used for commodity markets; for instance Dias and Carlos Rocha [9] price extendable oil options. Neftci and Santos [25] note that extendable bonds have stabilizing properties and "the embedded options work as a cushion and replicate the trading gains from hedging long-term bonds with interest rate derivatives." Recently, Koussis et al. [21] consider the problem of product development, which inherently contain extendable features, within the real option framework that generalizes the results of Longstaff [22]. Indeed the application of extendable features within the real option framework deserves further investigation.

The earliest work on financial derivatives that have this feature appears in Ananthanarayanan and Schwartz [1] and Brennan and Schwart [3] which display theoretical pricing for retractable and extendable bonds. Longstaff [22] discusses extendable options extensively and provides a closed-form solution for extendable options under the Black-Scholes model [2]. In recent years, Chung and Johnson [6] extend the work of Longstaff [22] to a general case where the holder or the writer can extend the option more than once, and derive a closed-form solution for $n$-extensions. While these studies are within the Black-Scholes framework, Gukhal [12] provides closed-form solutions for the extendable option with a jump feature based on the Merton jump diffusion model [23], and demonstrates that a compound option is a special case of the extendable option. Additionally, Peng and Peng [26] extend the study of Chung and Johnson [6] by deriving a value for an $n$-time extendable option with jumps, where the underlying asset price follows a fractional process, while Shevchenko [30] derives the price for extendable option for the case of an underlying asset that follows a geometric Brownian motion with time-dependent and volatility.

Given the underlying asset price $S$ and the initial strike price $K_{1}$, at a given initial maturity time $T_{1}$, the call option can be extended to time $T_{2}$ for a new strike price $K_{2}$ 
by paying an additional premium $A$. The payoff of the holder-extendable call option can be represented as follows:

$$
\max \left[0, S_{T_{1}}-K_{1}, C\left(S_{T_{1}}, K_{2}, T_{2}-T_{1}\right)-A\right],
$$

or similarly:

$$
\max \left[\max \left(S_{T_{1}}-K_{1}, 0\right), \max \left[C\left(S_{T_{1}}, K_{2}, T_{2}-T_{1}\right)-A, 0\right]\right],
$$

which indicates that at time $T_{1}$, the holder has to compare two risky payoffs and choose the largest payoff, where $C(S, K, t)$ is the price of a vanilla call option.

The holder of a holder-extendable call option has the right, but not the obligation to either let the option expire worthless; or to exercise the option; or to extend the option's maturity time. The choice region where the option is either exercised or extended is determined by solving for the critical asset values $s^{*}$ and $s^{* *}$ which may be obtained from the following equations:

$$
\begin{array}{r}
C\left(s^{*}, K_{2}, T_{2}-T_{1}\right)-A=0, \\
s^{* *}-K_{1}=C\left(s^{* *}, K_{2}, T_{2}-T_{1}\right)-A .
\end{array}
$$

Equation (3) has a unique solution $s^{*}=L$ that is bounded by $A \leq L \leq A+K_{2} e^{-r\left(T_{2}-T_{1}\right.}$. If $L \geq K_{1}$, then the call option is never extended; hence the holder receives $C\left(S_{T_{1}}, K_{1}, T_{1}\right)$. If $L<K_{1}$, then Equation (4) has a finite unique solution $s^{* *}=H$ when $A>K_{1}-K_{2} e^{-r\left(T_{2}-T_{1}\right)}$, where the call option is extended when $L<S_{T_{1}}<H$; exercised when $S_{T_{1}} \geq H$; and worthless when $S_{T_{1}} \leq L$. This is the usual case for a holder-extendable call option and is depicted in Figure 1, where the black line represents the payoff of $\max \left(S_{T_{1}}-K_{1}, 0\right)$, and the red line represents the payoff of $C\left(S_{T_{1}}, K_{2}, T_{2}-T_{1}\right)-A$. However, when $A \leq K_{1}-K_{2} e^{-r\left(T_{2}-T_{1}\right)}$, Equation (4) has no solution if $L<K_{1}$ where the call option is extended when $L<S_{T_{1}}$ and worthless when $L \geq S_{T_{1}} \cdot{ }^{1}$ Figure 2 illustrates this.

The fair price of an option whose price depends on its underlying asset price can be determined under the risk-neutral probability measure $\mathbb{Q}$, where the expected return on the risky asset is same as that on a risk-free investment in cash. Therefore, at maturity time $T$, the price of a holder-extendable call option $E C$ is computed as the discounted risk-neutral conditional expectation of its payoff (1) at a risk-free rate $r$ defined as follows:

$$
E C=e^{-r\left(T_{1}-t\right)} \mathbb{E}^{\mathbb{Q}} \max \left[0, S_{T_{1}}-K_{1}, C\left(S_{T_{1}}, K_{2}, T_{2}-T_{1}\right)-A\right],
$$

where $C$ is calculated in either the Black-Scholes framework [2], $C_{B S}$, or the Heston framework [14], $C_{H} \cdot{ }^{2}$ Hence, the analytical pricing solution for a holder-extendable call option in the Black-Scholes framework is given as follows [22, see]:

\footnotetext{
${ }^{1}$ The analysis of these conditions are also given in [30, 12].

${ }^{2}$ Note that the Heston vanilla call has a semi-analytic solution which is used in the Monte Carlo simulation under the Heston framework.
} 


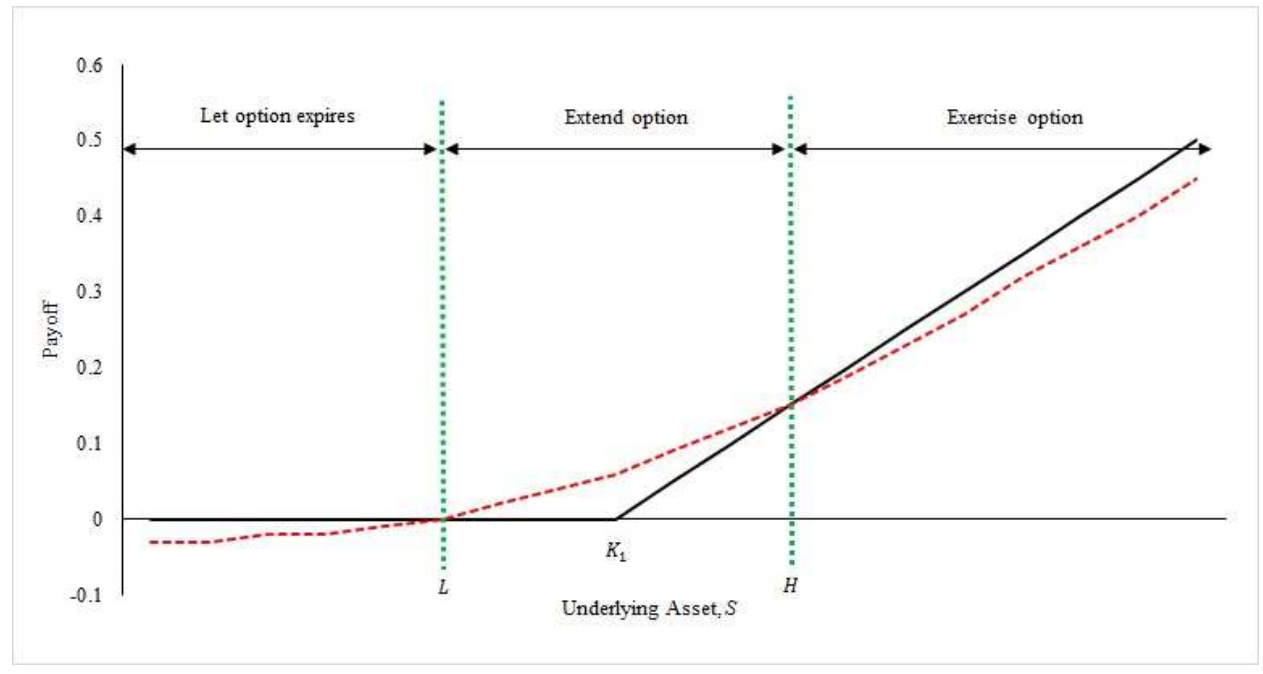

FIGURE 1. Holder-extendable call payoff at time $T_{1}$ when $H$ is finite and unique.

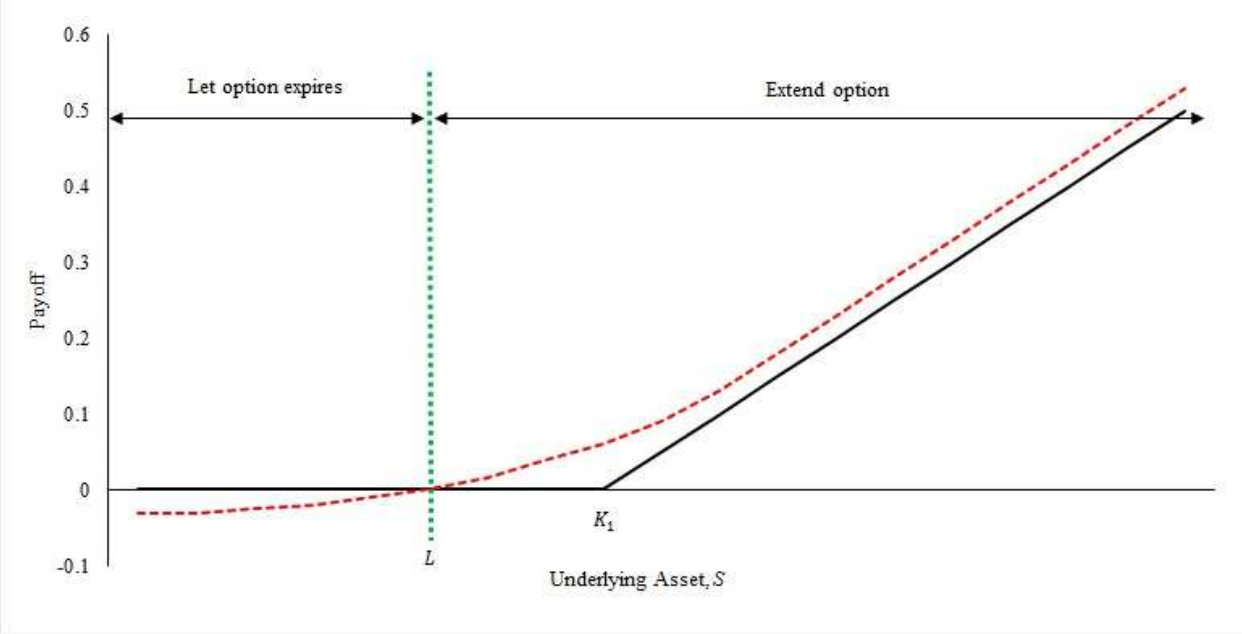

Figure 2. Holder-extendable call payoff at time $T_{1}$ when $H$ does not exist. 
THEOREM 1. Given underlying asset price $S$, initial maturity date $T_{1}$ and strike price $K_{1}$, the price of a holder-extendable call option whose maturity time may be extended to $T_{2}$ for an additional payment $A$ with a new strike price $K_{2}$, is as such:

$$
\begin{aligned}
& E C_{B S}\left(S_{t}, K_{1}, T_{1}, K_{2}, T_{2}, A\right) \\
& =C_{B} S\left(S_{t}, K_{1}, T_{1}\right) \\
& +\left[S_{t} M^{(2)}\left(a_{1}, b_{1},-\infty, c_{1} ; \rho\right)-K_{2} e^{-r\left(T_{2}-t\right)} M^{(2)}\left(a_{2}, b_{2},-\infty, c_{2} ; \rho\right)\right] \\
& -\left[S_{t} M\left(a_{1}, d_{1}\right)-K_{1} e^{-r\left(T_{1}-t\right)} M\left(a_{2}, d_{2}\right)\right]-A e^{-r\left(T_{1}-t\right)} M\left(a_{2}, b_{2}\right),
\end{aligned}
$$

where:

$$
\begin{array}{ll}
a_{1}=\frac{\ln \left(\frac{S_{t}}{H}\right)+\left(r+\frac{1}{2} \sigma^{2}\right)\left(T_{1}-t\right)}{\sigma \sqrt{T_{1}-t}}, & a_{2}=a_{1}-\sigma \sqrt{T_{1}-t} \\
b_{1}=\frac{\ln \left(\frac{S_{t}}{L}\right)+\left(r+\frac{1}{2} \sigma^{2}\right)\left(T_{1}-t\right)}{\sigma \sqrt{T_{1}-t}}, & b_{2}=b_{1}-\sigma \sqrt{T_{1}-t} \\
c_{1}=\frac{\ln \left(\frac{S_{t}}{K_{2}}\right)+\left(r+\frac{1}{2} \sigma^{2}\right)\left(T_{2}-t\right)}{\sigma \sqrt{T_{2}-t}}, & c_{2}=c_{1}-\sigma \sqrt{T_{2}-t} \\
d_{1}=\frac{\ln \left(\frac{S_{t}}{K_{1}}\right)+\left(r+\frac{1}{2} \sigma^{2}\right)\left(T_{1}-t\right)}{\sigma \sqrt{T_{1}-t}}, & d_{2}=d_{1}-\sigma \sqrt{T_{1}-t} \\
\rho=\sqrt{\frac{T_{1}-t}{T_{2}-t}} &
\end{array}
$$

and $M(a, b)$ is the cumulative probability of the standard normal density in the interval $[a, b]$, while $M^{(2)}(a, b, c, d ; \rho)$ is the cumulative probability of the standard bivariate normal density with correlation $\rho$ for the region $[a, b] \times[c, d]$.

The price of a holder-extendable call option (6) in Theorem 1 can be represented in terms of fewer univariate normal distributions by using the following identities:

$$
\begin{aligned}
M^{(2)}(a, b, c, d ; \rho) & =N^{(2)}(b, d ; \rho)-N^{(2)}(a, d ; \rho)-N^{(2)}(b, c ; \rho)+N^{(2)}(a, c ; \rho), \\
M^{(2)}(a, b,-\infty, d ; \rho) & =N^{(2)}(b, d ; \rho)-N^{(2)}(a, d ; \rho), \\
M(a, b) & =N(b)-N(a),
\end{aligned}
$$

where $N(\cdot)$ is the standard normal distribution and $N^{(2)}(\cdot, \cdot ; \rho)$ is the standard bivariate normal distribution with correlation $\rho$, which yields the following corollary.

COROLlary 1. The price of a holder-extendible call option with maturity $T_{1}$ and strike price $K_{1}$, whose maturity may be extended to $T_{2}$ with a new strike price $K_{2}$ by making 
an additional payment $A$ is:

$$
\begin{aligned}
& E C_{B S}\left(S_{t}, K_{1}, T_{1}, K_{2}, T_{2}, A\right) \\
& =C_{B S}\left(S_{t}, K_{1}, T_{1}\right)+S_{t} N^{(2)}\left(b_{1}, c_{1} ; \rho\right)-K_{2} e^{-r\left(T_{2}-t\right)} N^{(2)}\left(b_{2}, c_{2} ; \rho\right) \\
& -\left[S_{t} N^{(2)}\left(a_{1}, c_{1} ; \rho\right)-K_{2} e^{-r\left(T_{2}-t\right)} N^{(2)}\left(a_{2}, c_{2} ; \rho\right)\right] \\
& -A e^{-r\left(T_{1}-t\right)}\left[N\left(b_{2}\right)-N\left(a_{2}\right)\right],
\end{aligned}
$$

where $a_{1}, a_{2}, b_{1}, b_{2}, c_{1}$, and $c_{2}$ are as defined previously.

Equation (6) represents the price of a vanilla call option with strike price $K_{1}$ plus the non-negative value of the extension privilege. Hence, the holder-extendable call option is worth at least as much as its corresponding vanilla call option, and the holderextendable call option is worthless when letting $S=0$. Moreover, by letting $L=0$ and $H=\infty$ reduces the holder-extendable call option to a vanilla call option that is always extended which yields $C\left(S_{t}, K_{2}, T_{2}\right)$. Additionally, in Equation (3), by imposing $A=0$ yields $L=0$, and taking $H=K_{1}$, reduces the holder-extendable call option pricing formula (6) to a writer-extendable call option pricing formula.

In this study, we consider the problem of pricing holder-extendable call options under the Heston model [14], which is characterized by the following dynamics:

$$
\begin{aligned}
d S_{t} & =r S_{t} d t+\sqrt{v_{t}} S_{t} d W_{t, 1}, \\
d v_{t} & =\kappa\left(\theta-v_{t}\right) d t+\sigma_{0} \sqrt{v_{t}} d W_{t, 2},
\end{aligned}
$$

where $<d W_{t, 1}, d W_{t, 2}>=\rho d t, \kappa \geq 0$ is the speed of mean reversion, $\theta \geq 0$ is the mean level of variance, $\sigma_{0}>0$ is the volatility of the volatility, and $v_{t}$ follows a meanreverting square-root process [8]. It is convenient to write the equations above in terms of two independent Brownian motions $\left(\tilde{W}_{t, 1}, \tilde{W}_{t, 2}\right)$ such that:

$$
\begin{aligned}
& d S_{t}=r S_{t} d t+\sqrt{v_{t}} S_{t}\left(\sqrt{1-\rho^{2}} d \tilde{W}_{t, 1}+\rho d \tilde{W}_{t, 2}\right) \\
& d v_{t}=\kappa\left(\theta-v_{t}\right) d t+\sigma_{0} \sqrt{v_{t}} d \tilde{W}_{t, 2},
\end{aligned}
$$

or:

$$
d\left(\begin{array}{c}
S_{t} \\
v_{t}
\end{array}\right)=\left(\begin{array}{c}
r S_{t} \\
\kappa\left(\theta-v_{t}\right)
\end{array}\right) d t+\left(\begin{array}{cc}
\sqrt{v_{t}\left(1-\rho^{2}\right)} S_{t} & \rho \sqrt{v_{t}} S_{t} \\
0 & \sigma_{0} \sqrt{v_{t}}
\end{array}\right)\left(\begin{array}{c}
d \tilde{W}_{t, 1} \\
d \tilde{W}_{t, 2}
\end{array}\right),
$$

where $E\left[d \tilde{W}_{t, 1} d \tilde{W}_{t, 2}\right]=0$, i.e. $d \tilde{W}_{t, 1}$ is uncorrelated with $d \tilde{W}_{t, 2}$. In order to compare against the Black-Scholes formulation, the expected variance (given an initial variance $v_{0}$ ) over the life of an option of maturity $T$ is required [14]. Under the Heston dynamics this is given by Rouah [28]:

$$
\bar{v}(T)=\mathbb{E}^{\mathbb{Q}}\left[\int_{0}^{T} v_{t} d t \mid v_{0}\right]=\left(v_{0}-\theta\right)\left(\frac{1-e^{-\kappa T}}{\kappa}\right)+\theta T .
$$


According to Sophocleous et al. [31], the complexity of the model increases when stochastic volatility is incorporated. Hence, a numerical technique is used to price options with this additional feature. Numerical techniques may include Monte Carlo simulation and finite difference methods [7, 10]. The FFT technique in option pricing was introduced by Carr and Madan [5], and has since gained popularity in option pricing because its algorithm offers computational efficiency by employing the characteristic function of the log price which is known in closed-form for many models discussed in the literature [17, 27, 32, 33, 34]. In Ibrahim et al. [19], the FFT technique has been applied to price the holder-extendable call options in the BlackScholes environment, while in this study, we aim to apply the FFT technique to price the holder-extendable call options under the Heston model [14].

The remainder of the paper is organized as follows. Section 2 provides the characteristic functions and numerical solutions of extendable option by FFT technique. The extendable option is expressed as expectations of indicator functions and the inverse Fourier transform (iFT) is obtained for one- and two-dimensional FFT. We employ known closed-form representation of characteristic functions to use in the implementation of the FFT. The numerical results in Section 3 documents the effectiveness and efficiency of the proposed model against two benchmarks: Monte Carlo simulation (MCS) and finite difference methods (FDM). Section 4 concludes the paper.

\section{The Fast Fourier Transform (FFT)}

In this section, we implement the FFT technique to price an extendable option by expressing the payoff function as a difference of its expectations of indicator functions [4]. The FFT approach utilizes the characteristic function of the underlying asset price process. For the extendable option, the implementation involves a univariate and a bivariate characteristic functions under the risk-neutral measure $\mathbb{Q}$. The characteristic function is defined as follows:

Definition 1. Given two stochastic processes $X_{t}$ and $Y_{t}$ for $0 \leq t \leq T$, with density functions $q_{T}\left(X_{T}\right)$ and $q_{T}\left(Y_{T}\right)$, the characteristic function is the Fourier transform of its density function such that:

$$
\varphi\left(u_{1}\right)=\mathbb{E}^{\mathbb{Q}}\left(e^{i u_{1} X_{T}}\right),
$$

for a one-dimensional stochastic process, and:

$$
\varphi\left(u_{1}, u_{2}\right)=\mathbb{E}^{\mathbb{Q}}\left(e^{i u_{1} X_{T}}+e^{i u_{2} Y_{T}}\right),
$$

for a two-dimensional stochastic process where $u$ is the arbitraty real numbers and $i=\sqrt{-1}$ is the imaginary unit.

The following lemmas present the univariate characteristic function as provided in Heston [14], and the bivariate characteristic function that is obtained from the arguments presented in Griebsch and Wystup [11], under the Heston model. 
Lemma 1. Under the Heston model, a univariate characteristic function is as follows:

$$
\begin{aligned}
\varphi_{x_{T_{1}}}\left(u_{1}\right)= & \exp \left\{i u_{1}\left(x_{t}+r\left(T_{1}-t\right)+\frac{\rho}{\sigma_{0}}\left[-v_{0}-\kappa \theta\left(T_{1}-t\right)\right]\right)\right\} \\
& \times \exp \left[A\left(T_{1}-t, a\left(u_{1}\right), b\left(u_{1}\right)\right) v_{0}\right] \\
& \times \exp \left[B\left(T_{1}-t, a\left(u_{1}\right), b\left(u_{1}\right)\right)\right],
\end{aligned}
$$

where:

$$
\begin{aligned}
A(\tau, a, b) & =\frac{d a(u)\left(1+e^{-d \tau}\right)-[\kappa a(u)+2 b(u)]\left[1-e^{-d \tau}\right]}{2 d e^{-d \tau}+\left[\sigma_{0}^{2} a(u)-\kappa-d\right]\left[e^{-d \tau}-1\right]} \\
B(\tau, a, b) & =\frac{\kappa \theta}{\sigma_{0}^{2}}(\kappa-d) \tau+\frac{2 \kappa \theta}{\sigma_{0}^{2}} \ln \left[\frac{2 d}{2 d e^{-d \tau}+\left[\kappa+d-\sigma_{0}^{2} a(u)\right]\left[1-e^{-d \tau}\right]}\right], \\
a(u) & =i u \frac{\rho}{\sigma_{0}} \\
b(u) & =i u\left[-\frac{1}{2}+\kappa \frac{\rho}{\sigma_{0}}+\frac{1}{2} i u\left(1-\rho^{2}\right)\right] \\
d & =\sqrt{\kappa^{2}+2 \sigma_{0}^{2} b(u)} .
\end{aligned}
$$

Lemma 2. Under the Heston model, a bivariate characteristic function is as follows:

$$
\begin{aligned}
& \varphi_{x_{T_{1}}, x_{T_{2}}}\left(u_{1}, u_{2}\right) \\
& =\exp \left\{i u_{1}\left(x_{t}+r\left(T_{1}-t\right)+\frac{\rho}{\sigma_{0}}\left[-v_{0}-\kappa \theta\left(T_{1}-t\right)\right]\right)\right\} \\
& \times \exp \left\{i u_{2}\left(x_{t}+r\left(T_{2}-t\right)+\frac{\rho}{\sigma_{0}}\left[-v_{0}-\kappa \theta\left(T_{2}-t\right)\right]\right)\right\} \\
& \times \exp \left\{B\left(T_{2}-T_{1}, a\left(u_{2}\right), b\left(u_{2}\right)\right)+A\left(T_{1}-t, C(\tau, a, b), b\left(u_{1}+u_{2}\right)\right) v_{0}\right\} \\
& \times \exp \left\{B\left(T_{1}-t, C(\tau, a, b), b\left(u_{1}+u_{2}\right)\right)\right\},
\end{aligned}
$$

where:

$$
C(\tau, a, b)=a\left(u_{1}\right)+A\left(T_{2}-T_{1}, a\left(u_{2}\right), b\left(u_{2}\right)\right),
$$

and

$$
A(\tau, a, b), B(\tau, a, b), a(u), b(u), d
$$

are as defined in Lemma 1.

By the martingale property under the risk-neutral measure $\mathbb{Q}$, the holder-extendible call option as shown in Equation (5) can be valued at time $t$ using expectations of 
indicator-functions as follows:

$$
\begin{aligned}
& E C_{H}\left(S_{t}, K_{1}, T_{1}, K_{2}, T_{2}, A\right) \\
& =e^{-r\left(T_{1}-t\right)} \underbrace{\underbrace{\mathbb{Q}}\left[\left(e^{x_{T_{1}}}-e^{k_{1}}\right) \mathbf{1}_{\left\{x_{T_{1}}>h\right\}}\right]}_{I} \\
& +e^{-r\left(T_{2}-t\right)} \underbrace{\mathbb{E}^{\mathbb{Q}}\left[\left(e^{x_{T_{2}}}-e^{k_{2}}\right) \mathbf{1}_{\left\{x_{T_{1}} \geq l, x_{T_{2}} \geq k_{2}\right\}}\right]}_{I I} \\
& -e^{-r\left(T_{2}-t\right)} \underbrace{\mathbb{E}^{\mathbb{Q}\left[\left(e^{x_{T_{2}}}-e^{k_{2}}\right) \mathbf{1}_{\left\{x_{T_{1}} \geq h, x_{T_{2}} \geq k_{2}\right\}}\right]}}_{I V} \\
& -e^{-r\left(T_{1}-t\right)} \underbrace{\mathbb{E}^{\mathbb{Q}\left[e^{a} \mathbf{1}_{\left\{x_{T_{1}} \geq l\right\}}-e^{a} \mathbf{1}_{\left\{x_{T_{1}} \geq h\right\}}\right]},}_{I V}
\end{aligned}
$$

where $x_{t}=\ln S_{t}, k_{1}=\ln K_{1}, k_{2}=\ln K_{2}, l=\ln L, h=\ln H$ and $a=\ln A$. In integral form, Equation (16) can be written as:

$$
\begin{aligned}
& E C_{H}\left(S_{t}, K_{1}, T_{1}, K_{2}, T_{2}, A\right) \\
& =e^{-r\left(T_{1}-t\right)} \underbrace{\int_{h}^{\infty}\left(e^{x_{T_{1}}}-e^{k_{1}}\right) q\left(x_{T_{1}}\right) d x_{T_{1}}}_{I} \\
& +e^{-r\left(T_{2}-t\right)} \underbrace{\int_{l}^{\infty} \int_{k_{2}}^{\infty}\left(e^{x_{T_{2}}}-e^{k_{2}}\right) q\left(x_{T_{1}}, x_{T_{2}}\right) d x_{T_{2}} d x_{T_{1}}}_{I I} \\
& -e^{-r\left(T_{2}-t\right)} \underbrace{\int_{h}^{\infty} \int_{k_{2}}^{\infty}\left(e^{x_{T_{2}}}-e^{k_{2}}\right) q\left(x_{T_{1}}, x_{T_{2}}\right) d x_{T_{2}} d x_{T_{1}}}_{I I I} \\
& -e^{-r\left(T_{1}-t\right)}[\underbrace{e^{a} \int_{l}^{\infty} q\left(x_{T_{1}}\right) d x_{T_{1}}}_{I V}-\underbrace{e^{a} \int_{h}^{\infty} q\left(x_{T_{1}}\right) d x_{T_{1}}}_{V}],
\end{aligned}
$$

where $q(\cdot)$ is the conditional density function of the random value $x_{T_{1}}$, and $q(\cdot, \cdot)$ is the joint conditional density function of the random variable $x_{T_{1}}$ and $x_{T_{2}}$ for a given value $x_{t}$.

Employing a similar approach to Carr and Madan [5], we implement FFT on terms $I-V$. To avoid repetition, we only consider term $V$ for the univariate case, and term $I I I$ 
for the bivariate case. First we multiply terms $V$ and $I I I$ by an exponentially decaying term so that it is square-integrable, and we define the damped integral as follows:

$$
\begin{aligned}
V^{D}(h) & =e^{\alpha_{1} h} V(h), \\
I I I^{D}\left(h, k_{2}\right) & =e^{\alpha_{1} h+\alpha_{2} k_{2}} I I I\left(h, k_{2}\right),
\end{aligned}
$$

for $\alpha_{1}, \alpha_{2}>0$. Then we apply Fourier transform:

$$
\begin{aligned}
\psi\left(u_{1}\right) & =\int_{-\infty}^{\infty} e^{i u_{1} h} V^{D}(h) d h, \\
\psi\left(u_{1}, u_{2}\right) & =\int_{-\infty}^{\infty} \int_{-\infty}^{\infty} e^{i u_{1} h+i u_{2} k_{2}} I I I^{D}\left(h, k_{2}\right) d k_{2} d h,
\end{aligned}
$$

where the Fourier transform $\psi$ is available in closed-form in terms of the characteristic function $\varphi$ as follows:

$$
\begin{aligned}
\psi\left(u_{1}\right) & =\frac{\varphi_{x_{T_{1}}}\left(u_{1}-i \alpha_{1}\right)}{i u_{1}+\alpha_{1}}, \\
\psi\left(u_{1}, u_{2}\right) & =\frac{\varphi_{x_{T_{1}}, x_{T_{2}}}\left(u_{1}-i \alpha_{1}, u_{2}-i\left(\alpha_{2}+1\right)\right)}{\left(i u_{1}+\alpha_{1}\right)\left(i u_{2}+\alpha_{2}\right)\left(1+i u_{2}+\alpha_{2}\right)} .
\end{aligned}
$$

Using IFT, we recover terms $V$ and $I I I$ as such:

$$
\begin{aligned}
V(h) & =\frac{e^{-\alpha_{1} h}}{2 \pi} \int_{-\infty}^{\infty} e^{-i u_{1} h} \psi\left(u_{1}\right) d u_{1}, \\
I I I\left(h, k_{2}\right) & =\frac{e^{-\alpha_{1} h-\alpha_{2} k_{2}}}{(2 \pi)^{2}} \int_{-\infty}^{\infty} \int_{-\infty}^{\infty} e^{-i u_{1} h-i u_{2} k_{2}} \psi\left(u_{1}, u_{2}\right) d u_{2} d u_{1} .
\end{aligned}
$$

The integrals in Equations (18) and (19) are evaluated by numerical approximation using the trapezoidal rule and FFT given as follows:

$$
\begin{aligned}
V(h) & \approx \frac{e^{-\alpha_{1} h}}{2 \pi} \sum_{j=0}^{N-1} e^{-i u_{1, j} h} \psi\left(u_{1, j}\right) \Delta_{1}, \\
I I I\left(h, k_{2}\right) & \approx \frac{e^{-\alpha_{1} h-\alpha_{2} k_{2}}}{(2 \pi)^{2}} \sum_{j=0}^{N-1} \sum_{m=0}^{N-1} e^{-i u_{1, j} h-i u_{2, m} k_{2}} \psi\left(u_{1, j}, u_{2, m}\right) \Delta_{2} \Delta_{1},
\end{aligned}
$$

where $\Delta_{1}$ and $\Delta_{2}$ denote the distance between the points of the integration grid, and $u_{1, j}=\left(j-\frac{N}{2}\right) \Delta_{1}, \quad u_{2, m}=\left(m-\frac{N}{2}\right) \Delta_{2}$, for $j, m=0, \ldots, N-1$ (where $N=2^{n}, n \in$ $\mathbb{N})$. Then we define a grid of size $N \times N$, by $H_{2}=\left\{\left(h_{u}, k_{2, p}\right) \mid 0 \leq u, p \leq N-1\right\}$ 
with $\omega_{1}, \omega_{2}>0$ denoting the distance between the logarithmic critical prices and the logarithmic strike prices, respectively, as follows: $h_{u}=\left(u-\frac{N}{2}\right) \omega_{1}, \quad k_{2, p}=$ $\left(p-\frac{N}{2}\right) \omega_{2}$, and then evaluate by the following sum:

$$
\begin{aligned}
Z(h) & =\sum_{j=0}^{N-1} e^{-i u_{1, j} h} \psi\left(u_{1, j}\right), \\
Z\left(h, k_{2}\right) & =\sum_{j=0}^{N-1} \sum_{m=0}^{N-1} e^{-i u_{1, j} h-i u_{2, m} k_{2}} \psi\left(u_{1, j}, u_{2, m}\right) .
\end{aligned}
$$

Choosing $\omega_{1} \Delta_{1}=\frac{2 \pi}{N}$ and $\omega_{2} \Delta_{2}=\frac{2 \pi}{N}$, yields the following values of sum on $H_{2}$ :

$$
\begin{aligned}
Z\left(h_{u}\right) & =\sum_{j=0}^{N-1} e^{-i u_{1, j} h_{u}} \psi\left(u_{1, j}\right) \\
& =(-1)^{u} \sum_{j=0}^{N-1} e^{-i \frac{2 \pi}{N} j u}\left[(-1)^{j} \psi\left(u_{1, j}\right)\right] \\
Z\left(h_{u}, k_{2, p}\right) & =\sum_{j=0}^{N-1} \sum_{m=0}^{N-1} e^{-i u_{1, j} h_{u}-i u_{2, m} k_{2, p}} \psi\left(u_{1, j}, u_{2, m}\right) \\
& =(-1)^{u+p} \sum_{j=0}^{N-1} \sum_{m=0}^{N-1} e^{-i \frac{2 \pi}{N} j u-i \frac{2 \pi}{N} m p}\left[(-1)^{j+m} \psi\left(u_{1, j}, u_{2, m}\right)\right] .
\end{aligned}
$$

On that account, Equations (20) and (21) are computed via FFT by taking the input array, respectively, as follows:

$$
\begin{aligned}
X[j] & =(-1)^{j} \psi\left(u_{1, j}\right), \\
X[j, m] & =(-1)^{j+m} \psi\left(u_{1, j}, u_{2, m}\right),
\end{aligned}
$$

for $j, m=0, \ldots, N-1$. Therefore, the result is an approximation of term $V(h)$ at $N \times 1$ different logarithmic critical prices $h$, and term $I I I\left(h, k_{2}\right)$ at $N \times N$ different logarithmic critical prices $h$ and logarithmic strike prices $k_{2}$, specified by the following:

$$
\begin{aligned}
V\left(h_{u}\right) & \approx \frac{e^{-\alpha_{1} h_{u}}}{2 \pi} Z\left(h_{u}\right) \Delta_{1}, \\
I I I\left(h_{u}, k_{2, p}\right) & \approx \frac{e^{-\alpha_{1} h_{u}-\alpha_{2} k_{2, p}}}{(2 \pi)^{2}} Z\left(h_{u}, k_{2, p}\right) \Delta_{2} \Delta_{1},
\end{aligned}
$$

for $0 \leq u, p \leq N-1$. Following similar procedures as shown above, analogous results are obtained for terms $I$ and $I V$ for the univariate case, and term $I I$ for the bivariate case. 


\section{Numerical example}

In this section, we analyze the pricing of extendable options using the model from Section 2. We first evaluate option prices using FFT. Then we compare the accuracy and computational time of the pricing under the Heston model with two benchmark prices determined via Monte Carlo simulation (MCS) and the finite difference method (FDM). We adopt two commonly employed calibration errors, the absolute relative error (ARE) and the root-mean-squared error (RMSE). The ARE is defined for each initial stock price $j$, by:

$$
A R E_{j}=\left|\left(\frac{\widehat{E C}_{j}}{E C_{j}}-1\right)\right|,
$$

and the RMSE is defined by:

$$
R M S E=\sqrt{\frac{1}{n_{S}} \sum_{j=1}^{n_{S}}\left|\widehat{E C}_{j}-E C_{j}\right|^{2}},
$$

where the sum is over the number $n_{S}(=5)$, of initial stock values, $\widehat{E C}$ is the estimate price obtained via FFT, and $E C$ is the exact price determined by MCS or FDM. ${ }^{1}$ For the implementation of the FFT technique it is convenient to allow the outer and the inner sum of Equation (21) to have different $N=N_{1}$ and $N_{2}\left(<2^{12}\right)$ respectively. The FFT prices are sensitive to the choice of $\Delta_{1}, \Delta_{2}, \alpha_{1}, \alpha_{2}, N_{1}$ and $N_{2}$ [15]. Hence, adapting the methodology employed by Hurd and Zhou [18], we determine an appropriate choice of these parameters by minimizing the average of the absolute value of the log price differences:

$$
\operatorname{Err}=\frac{1}{n_{S}} \sum_{j=1}^{n_{S}}\left|\log \left(\widehat{E C}_{j}\right)-\log \left(E C_{j}\right)\right| .
$$

A two step approach is taken to optimize the FFT parameters. First $\alpha_{1}$ and $\Delta_{1}$ are fixed at 0.75 and 0.1 respectively, which are reasonable parameters [15]. The outputs for $\alpha_{2}$ and $\Delta_{2}$ are then employed (together with $\alpha_{1}$ and $\Delta_{1}$ ), as initial inputs to minimize Err. This is repeated for different $N_{1}$ and $N_{2}$. In all that follows, these parameters are determined to be: $N_{1}=2^{6}, N_{2}=2^{4}, \Delta_{1}=0.1637, \Delta_{2}=0.0166, \alpha_{1}=0.7496$ and $\alpha_{2}=0.7502$. In the MCS approach, we use 100, 000 simulations each of 1000 steps (following Hurd and Zhou [18], no variance reduction was employed). Moreover, the FDM is applied over a three-dimension grid, $50 \times 50 \times 7000$ (stock price, variance and time to maturity).

Table 1 lists the other parameter values used in the computation. The Heston parameters, tabulated in Table 2, are sourced from Table 5 in Moyaert and Petitjean [24] where these values are calibrated from the market prices of Eurostoxx 50 index

\footnotetext{
${ }^{1}$ The computations were implemented in MATLAB and conducted on an Intel (R) Core(TM) i7-7700 CPU @ $3.60 \mathrm{GHz}$ machine running under Windows 10 with 12GB RAM and 64-bit operating system.
} 
TABLE 1. Inputs to price the extendable options

\begin{tabular}{lr}
\hline Input & Value \\
\hline initial stock prices, $S$ & $0.8,0.85,0.9,0.95,1.0$ \\
initial strike price, $K_{1}$ & 0.9 \\
initial expiration time, $T_{1}$ & 1 \\
extended strike price, $K_{2}$ & 0.95 \\
extended expiration time $T_{2}$ & 2 \\
risk-free rate, $r$ & 0.02 \\
premium, $A$ & 0.03 \\
\hline
\end{tabular}

TABLE 2. Heston parameters

\begin{tabular}{lr}
\hline Input & Value \\
\hline instantaneous volatility, $\sqrt{v}$ & 0.33 \\
long run volatility, $\sqrt{\theta}$ & 0.28 \\
mean reversion rate, $\kappa$ & 3.15 \\
volatility of variance, $\sigma_{0}$ & 0.76 \\
correlation parameter, $\rho$ & -0.81 \\
\hline
\end{tabular}

options during the sub-prime crisis. With the parameter values from Tables 1 and 2, we solve for the critical prices using a root-search algorithm such as the NewtonRaphson method [20] and obtain $L=0.7946$ and $H=1.0753$. The comparison of holder-extendable call prices are tabulated in Table 3. Our numerical examples demonstrate that the computational time difference is significant as FFT takes $50.03 \mathrm{~s}$ to produce five holder-extendable call prices including optimizing the FFT parameters, while MCS and FDM take $892.68 s$ and $126.49 s$ respectively, to produce five holderextendable call prices.

In Table 4, the ARE and the RMSE for the holder-extendable call option priced using the FFT under the Heston model, compared to MCS and FDM, is presented. Compared to the MCS and FDM, the ARE and the RMSE indicate better model performance of the FFT, and the errors obtained for the FFT are generally close to those for non-extendable options [15]. Finally in Table 5, we document the prices of a vanilla call option and a holder-extendable call option under the Black-Scholes [2] and Heston [14] models. The volatility input for the Black-Scholes is given by $\sqrt{\bar{v}(T)}$ in Equation (13) where for the vanilla call, $T=T_{1}$, and for the holder-extendable call, $T=T_{2}$, leading to Black-Scholes annualized volatilities of $29.61 \%$ and $28.85 \%$ respectively.

It can be seen that for out-of-the money options the Black-Scholes price is greater 
TABLE 3. Holder-extendable call option prices under Heston model: FFT, MCS and FDM

\begin{tabular}{|c|c|cc|}
\hline$S$ & FFT & FDM & $\begin{array}{c}\text { MCS } \\
(95 \% \text { confidence interval })\end{array}$ \\
\hline 0.8 & 0.0646 & 0.0648 & $\begin{array}{c}0.0646 \\
(0.0641,0.0651)\end{array}$ \\
\hline 0.85 & 0.0916 & 0.0910 & $\begin{array}{c}0.0911 \\
(0.0904,0.0918)\end{array}$ \\
\hline 0.9 & 0.1219 & 0.1208 & 0.1216 \\
& & & $(0.1208,0.1224)$ \\
\hline 0.95 & 0.1550 & 0.1545 & 0.1550 \\
& & & $(0.1540,0.1560)$ \\
\hline 1.0 & 0.1902 & 0.1909 & 0.1913 \\
& & & $(0.1902,0.1924)$ \\
\hline
\end{tabular}

TABLE 4. ARE and RMSE (in \%) for pricing holder-extendable call option under Heston model: FFT vs MCS and FDM

\begin{tabular}{|c|cc|}
\hline$S$ & MCS & FDM \\
\hline 0.8 & 0.00 & 0.31 \\
0.85 & 0.55 & 0.66 \\
0.9 & 0.25 & 0.91 \\
0.95 & 0.00 & 0.32 \\
1.0 & 0.58 & 0.37 \\
\hline RMSE & 0.05568 & 0.06856 \\
\hline
\end{tabular}

than the Heston price, and vice versa for in-the-money options for both vanilla calls and holder-extendable calls $[2,14]$. This is well-known for vanilla calls when $\rho<0$ [28] and it is also the case for holder-extendable calls (whether priced by FFT, MCS or FDM) because the distribution of the logarithmic asset prices is negatively skewed when $\rho<0$, producing a heavier left tail of the distribution. Moreover, the BlackScholes implied volatility exhibit larger curvature than the Heston implied volatility.

From Table 5, the extension privilege is higher under the Heston model than under the Black-Scholes model. Figures 3, 4 and 5 illustrate the changes in the values of the extension privilege under the Heston model when $v_{0}, \sigma$ and $T_{2}$ increases, respectively. It can be observed that the extension privilege increases as the values of $v_{0}, \sigma$ and $T_{2}$ increases. 
TABLE 5. Vanilla and holder-extendable call prices under Black-Scholes and Heston models

\begin{tabular}{|c|ccc|ccc|}
\hline \multirow{2}{*}{$S$} & \multicolumn{3}{|c|}{ Black-Scholes } & \multicolumn{3}{c|}{ Heston } \\
& Vanilla & Holder-extendable & Extension privilege & Vanilla & Holder-extendable & Extension privilege \\
\hline 0.8 & 0.0632 & 0.0721 & 0.0089 & 0.0494 & 0.0656 & 0.0162 \\
0.85 & 0.0866 & 0.0962 & 0.0096 & 0.0755 & 0.0918 & 0.0163 \\
0.9 & 0.1140 & 0.1238 & 0.0098 & 0.1071 & 0.1216 & 0.0145 \\
0.95 & 0.1451 & 0.1548 & 0.0097 & 0.1416 & 0.1545 & 0.0129 \\
1.0 & 0.1794 & 0.1888 & 0.0094 & 0.1796 & 0.1908 & 0.0112 \\
\hline
\end{tabular}

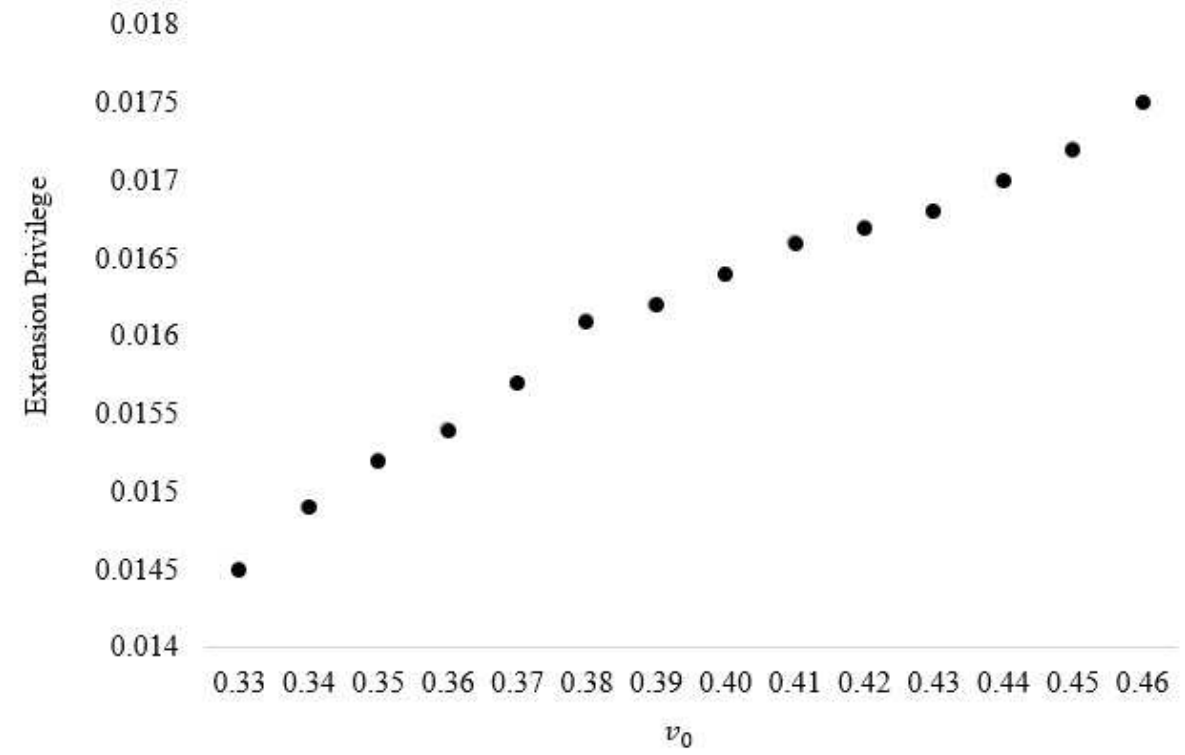

FIgURE 3. Extension privilege at time $T_{1}$ with different $v_{0}$. 


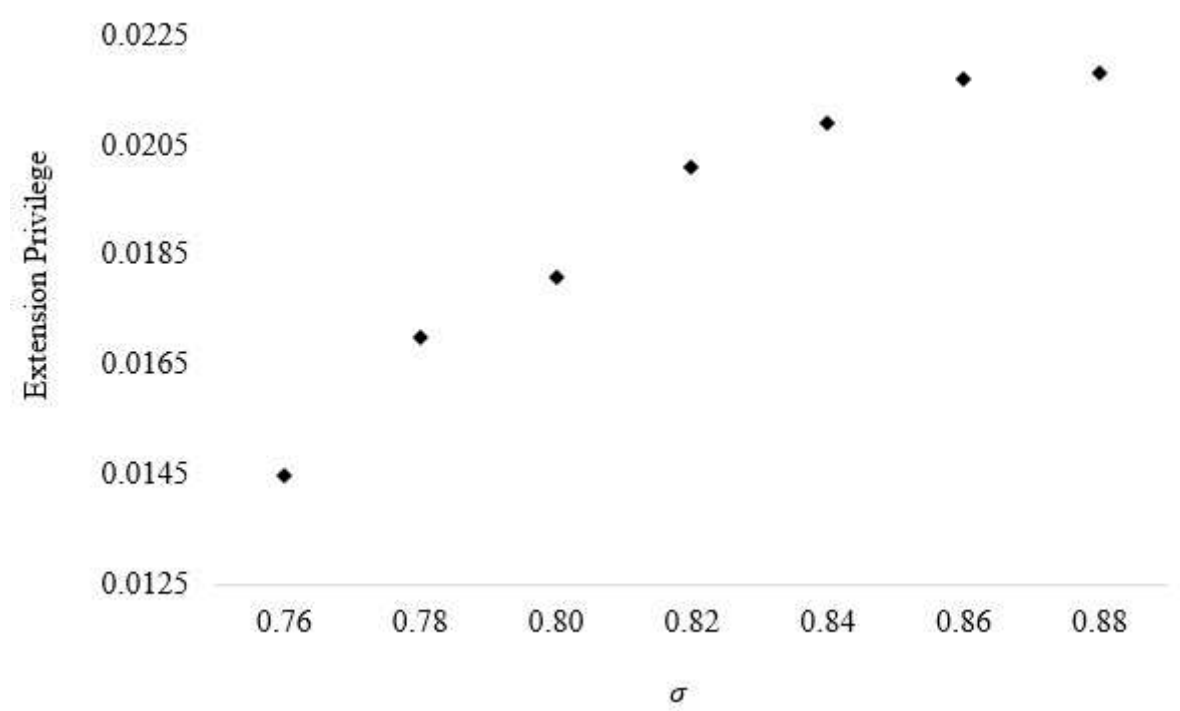

Figure 4. Extension privilege at time $T_{1}$ with different $\sigma$.

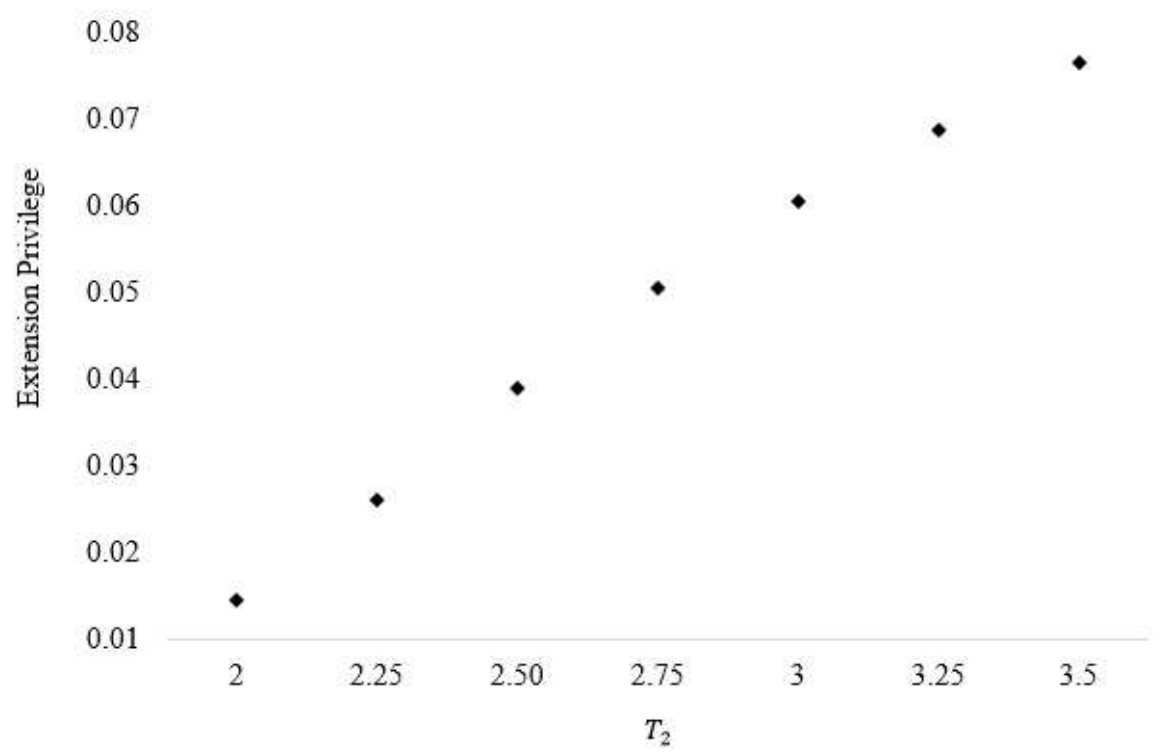

FIGURE 5. Extension privilege at time $T_{1}$ with different $T_{2}$. 


\section{Conclusion}

This paper considers the pricing of holder-extendable call options under the Heston dynamics using FFT and compared with the MCS and FDM benchmarks. The FFT pricing formula is expressible as a finite sum of expectations of indicator functions where the partition employs the two critical values introduced by Longstaff [22]. The evaluation of the expectations involve one-dimensional and two-dimensional Fourier transforms via the corresponding univariate and bivariate characteristic functions. Under the Heston model, there exist closed-form solutions of the characteristic functions; hence with comparison to MCS and explicit FDM, the application of the FFT yields significant computational savings, typically at least an order of magnitude. We also observe that overall the Heston model performs better than the Black-Scholes in pricing holder-extendable call options.

In addition to stochastic volatility, this study can be further developed by incorporating jumps with stochastic volatility together with stochastic interest rates in the spirit of Santa-Clara and Yan [29], and by implementing other optimization strategy. Finally, the use of extendable options in problem involving real options may lead to fruitful investigations.

\section{Acknowledgements}

We would like to thank the anonymous referees for their valuable comments and suggestions. We also gratefully acknowledge the contribution of the late Dr. Nick Constantinou to this paper. The author S.N.I. Ibrahim acknowledges the financial support from Ministry of Higher Education Malaysia and Universiti Putra Malaysia through FRGS (02-01-14-1464FR) and Geran Putra (GP/2017/9570100). The author A. Diaz-Hernandez thanks Universidad Anahuac Mexico-Norte and University of Essex for the financial support for a month-long visit for research.

\section{References}

[1] A. L. Ananthanarayanan and E. S. Schwartz. 'Retractable and extendible bond: The Canadian experience'. The Journal of Finance 35 (1) (1980), 31-47.

[2] F. Black and M. Scholes. 'The pricing of options and corporate liabilities'. Journal of Political Economy 81 (3) (1973), 637-654.

[3] M. J. Brennan and E. S. Schwartz. 'Savings bonds, retractable bonds and callable bonds'. Journal of Financial Economics 5 (1977), 67-88.

[4] P. W. Buchen. 'The pricing of dual-expiry exotics'. Quantitative Finance 4 (2004), 101-108.

[5] P. Carr and D. Madan. 'Option valuation using the fast Fourier transform'. Journal of Computational Finance 2 (4) (1999), 61-73.

[6] Y. P. Chung and H. Johnson. 'Extendible options: The general case'. Finance Research Letters 8 (2011), 15-20.

[7] R. Cont and E. Voltchkova. 'A finite difference scheme for option pricing in jump diffusion and exponential lévy models'. SIAM Journal on Numerical Analysis 43 (4) (2005), 15961626.

[8] J. C. Cox, J. E. Ingersoll, and S. A. Ross. 'A theory of the term structure of interest rate'. Econometrica 53 (2) (1985), 385-407.

[9] M. A. G. Dias and K. M. C. Rocha. 'Petroleum concenssions with extendible options using mean reversion with jumps to model oil prices', 1999. Working Paper, IPEA, Brazil. 
[10] P. Glasserman. Monte Carlo methods in financial engineering (Springer-Verlag New York, 2004).

[11] S. A. Griebsch and U. Wystup. 'On the valuation of fader and discrete barrier options in Heston's stochastic volatility model'. Quantitative Finance 11 (2011), 693-709.

[12] C. R. Gukhal. 'The compound option approach to American options on jump-diffusion'. Journal of Economic Dynamics and Control 28 (2004), 2055-2074.

[13] S. Hauser and B. Lauterbach. 'Empirical tests of the Longstaff extendible warrant model'. Journal of Empirical Finance 3 (1996), 1-14.

[14] S. L. Heston. 'A closed-form solution for options with stochastic volatility with applications to bond and currency options'. The Review of Financial Studies 6 (2) (1993), 327-343.

[15] A. Hirsa. Computational Methods in Finance (Chapman and Hall, 2013).

[16] J. S. Howe and P. Wei. 'The valuation effects of warrant extensions'. The Journal of Finance 48 (1) (1993), 305-314.

[17] J. Huang, W. Zhu, and X. Ruan. 'Fast Fourier transform based power option pricing with stochastic interest rate, volatility, and jump intensity'. Journal of Applied Mathematics 2013 (2013), 1-7.

[18] T. R. Hurd and Z. Zhou. 'A Fourier transform method for spread option pricing'. SIAM Journal on Financial Mathematics 1 (1) (2010), 142-157.

[19] S. N. I. Ibrahim, J. G. O'Hara, and N. Constantinou. 'Pricing extendible options using the fast fourier transform'. Mathematical Problems in Engineering 2014 (2014), 1-7.

[20] J. Kiusalaas. Numerical methods in engineering with python (Cambridge University Press, 2012), Second edition.

[21] N. Koussis, S. H. Martzoukos, and L. Trigeorgis. 'Multi-stage product development with exploration, value-enhancing, preemptive and innovation options'. Journal of Banking and Finance 37 (2013), 174-190.

[22] F. A. Longstaff. 'Pricing options with extendible maturities: Analysis and applications'. The Journal of Finance 45 (3) (1990), 935-957.

[23] R. C. Merton. 'Theory of rational option pricing'. The Bell Journal of Economics and Management Science 4 (1) (1973), 141-183.

[24] T. Moyaert and M. Petitjean. 'The performance of popular stochastic volatility option pricing models during the subprime crisis'. Applied Financial Economics 21 (14) (2011), 1059-1068.

[25] S. N. Neftci and A. O. Santos. 'Puttable and extendible bonds: Developing interest rate derivatives for emerging markets'. Working paper WP/03/201, International Monetary Fund, 2003.

[26] B. Peng and F. Peng. 'Pricing extendible option under jump-fraction process'. Journal of East China Normal University (Natural Science) 2012 (3) (2012), 30-40.

[27] E. Pillay and J. G. O'Hara. 'FFT based option pricing under a mean reverting process with stochastic volatility and jumps'. Journal of Computational and Applied Mathematics 235 (12) (2011), 3378-3384.

[28] F. D. Rouah. The Heston Model and its Extensions in Matlab and C\# (John Wiley \& Sons, New Jersey, 2013).

[29] P. Santa-Clara and S. Yan. 'Crashes, volatility, and the equity premium: Lessons from s\&p 500 options'. Review of Economics and Statistics 92 (2) (2010), 435451.

[30] P. V. Shevchenko. 'Holder-extendible European option: corrections and extensions'. The ANZIAM Journal 56 (4) (2015), 359-372.

[31] C. Sophocleous, J. G. O'Hara, and P. G. L. Leach. 'Algebraic solution of the Stein-Stein model for stochastic volatility'. Communications in Nonlinear Science and Numerical Simulation 16 (4) (2011), 17521759.

[32] S. Zhang and L. Wang. 'A fast Fourier transform technique for pricing European options with stochastic volatility and jump risk'. Mathematical Problems in Engineering 2012 (2012), 1-17.

[33] S. Zhang and L. Wang. 'Fast Fourier transform option pricing with stochastic interest rate, stochastic volatility and double jumps'. Applied Mathematics and Computation 219 (23) (2013), 1092810933.

[34] S. Zhang and L. Wang. 'A fast numerical approach to option pricing with stochastic interest rate, 
stochastic volatility and double jumps'. Communications in Nonlinear Science and Numerical Simulation 18 (7) (2013), 18321839. 